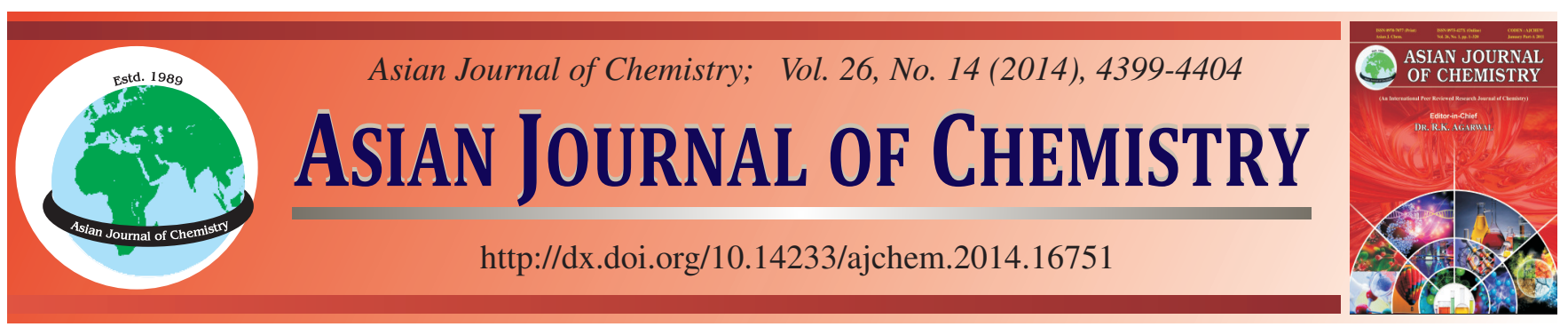

\title{
Two-Step Synthesis of Some Novel Monoindolylmaleimides
}

Jian Zhong ${ }^{\dagger}$, Ping JiA ${ }^{\dagger}$, Shan Xu, Liang Han, Yujing Li, Jianrong Gao and Qing Ye ${ }^{*}$

College of Chemical Engineering and Materials Science, Zhejiang University of Technology, Hangzhou 310014, P.R. China

*Corresponding author: E-mail: yeqing1975@zjut.edu.cn; yeqing1975@yeah.net

$\dagger$ These authors contributed equally

Received: 25 November 2013;

Accepted: 16 January 2014;

Published online: 5 July 2014;

AJC-15484

Monoindolylmaleimides have been identified as potent glycogen synthase kinase- $3 \beta$ inhibitors. In this paper, a convenient methodology for the preparation of some new monoindolylmaleimides was developed by microwave assisted condensation of substituted monochloromaleimides with $\mathrm{N}$-containing heterocycles followed by ammonolysis in good overall yield (up to $80 \%$ ). The products were characterized by ${ }^{1} \mathrm{H}$ NMR and ESI-MS.

Keywords: Monindolylmaleimides, GSK-3 $\beta$ inhibitors, Microwave assisted condensation, Microwave assisted ammonolysis.

\section{INTRODUCTION}

Monoindolylmaleimides such as imidazo[1,2-a]pyridinylindolylmaleimides, indazolyl-indolylmaleinides and benzofuranylindolylmaleimides have been identified as potent inhibitors of Glycogen synthase kinase- $3 \beta(\mathrm{GSK}-3 \beta)^{1-3}$, which are useful for the treatment of diseases such as cancer, diabetes type-2, chronic inflammatory processes, stroke, bipolar disorders and Alzheimer's disease ${ }^{4-8}$. These compounds were generally synthesized via condensation of a substituted indolyl-3-glyoxylate and a heteroaryl-3-acetamide using potassium tert-butoxide as a base $\mathrm{e}^{1-3}$. In former, we have found that the traditional synthetic route was not suitable for some kinds of monoindolylmaleimides such as imidazolylindolyl-maleimides, 1,3,4- triazolylindolylmaleimides and benzisoxazolylindolylmaleimides, suffered from poor yields, rigid reaction conditions and difficulty to obtain pure products ${ }^{9,10}$. Here we report a convenient method to synthesize some novel monoindolylmaleimides through microwave assisted condensation and ammonolysis reaction by using substituted monochloroindolylmaleimides and $\mathrm{N}$-containing heterocycles as the raw materials (Scheme-I).

\section{EXPERIMENTAL}

The reagents were obtained from commercial sources. DMF, MeCN were dried with $\mathrm{CaH}_{2}$ and toluene was dried with Na. Microwave reactions were carried out using a CEM

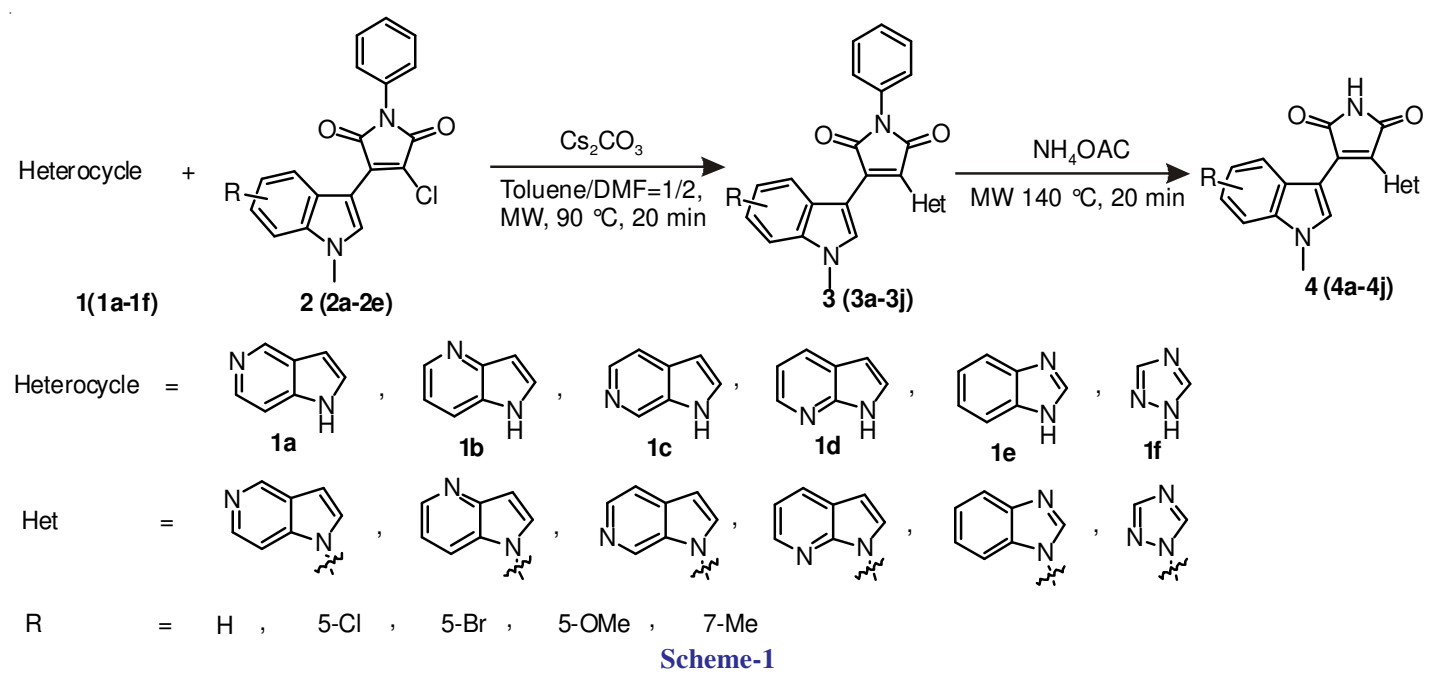


Discover instrument (CEM Corporation), in a $10 \mathrm{~mL}$ glass tube (CEM designed $10 \mathrm{~mL}$ pressure-rated reaction vial). Temperature was monitored by an infrared monitoring system. Nuclear magnetic resonance spectra were recorded on Bruker Avance III $500 \mathrm{MHz}$ and chemical shifts are expressed in ppm using TMS as an internal standard. ESI (positive) was recorded on an Esquire-LC-00075 spectrometer.

General procedure for the synthesis of 3a-3j: A mixture of substituted monochloromaleimide $(0.15 \mathrm{mmol})$, nitrogencontaining heterocycle $(0.18 \mathrm{mmol}), \mathrm{Cs}_{2} \mathrm{CO}_{3}(0.3 \mathrm{mmol})$ and mixed solvent $(3 \mathrm{~mL}$, toluene/DMF $=1 / 2$, v/v) was heated by microwave irradiation at $90{ }^{\circ} \mathrm{C}$ for $20 \mathrm{~min}$. After cooling, the resulted mixture was poured into water $(20 \mathrm{~mL})$ and extracted with ethyl acetate $(3 \times 20 \mathrm{~mL})$. The organic phase was combined, washed with brine $(3 \times 60 \mathrm{~mL})$, dried over $\mathrm{Na}_{2} \mathrm{SO}_{4}$ and concentrated in vасио. The residue was purified by flash column chromatography on silica gel using ethyl acetate/petroleum ether $(1 / 2, v / v)$ as eluent to afford compound $\mathbf{3} \mathbf{a}-\mathbf{3} \mathbf{j}^{11,12}$.

3-(1-Methyl-1H-indol-3-yl)-1-phenyl-4-(1H-pyrrolo[3,2-c]pyridin-1-yl)-1H-pyrrole-2,5-dione (3a): Orange solid, m.p. > $250{ }^{\circ} \mathrm{C} ;{ }^{1} \mathrm{H}$ NMR (500 MHz, DMSO-d $\left.d_{6}\right) \delta 8.30$ (dd, $J=4.6,1.3 \mathrm{~Hz}, 1 \mathrm{H}), 8.24$ (s, 1H), 7.89 (d, $J=3.5 \mathrm{~Hz}$, $1 \mathrm{H}), 7.60-7.52(\mathrm{~m}, 4 \mathrm{H}), 7.50(\mathrm{~d}, J=8.3 \mathrm{~Hz}, 1 \mathrm{H}), 7.48-7.42$ $(\mathrm{m}, 2 \mathrm{H}), 7.05(\mathrm{t}, J=7.7 \mathrm{~Hz}, 1 \mathrm{H}), 6.96-6.89(\mathrm{~m}, 2 \mathrm{H}), 6.57(\mathrm{t}$, $J=7.5 \mathrm{~Hz}, 1 \mathrm{H}), 6.00(\mathrm{~d}, J=8.1 \mathrm{~Hz}, 1 \mathrm{H}), 3.93$ (s, 3H); EI-MS: $419[\mathrm{M}+1]^{+}$.

3-(5-Chloro-1-methyl-1H-indol-3-yl)-1-phenyl-4-(1Hpyrrolo[3,2-c]pyridin-1-yl)-1H-pyrrole-2,5-dione (3b): Orange solid, m.p. 107-109 ${ }^{\circ} \mathrm{C} ;{ }^{1} \mathrm{H}$ NMR $(500 \mathrm{MHz}, \mathrm{DMSO}-$ $\left.d_{6}\right) \delta 8.32(\mathrm{dd}, J=4.6,1.3 \mathrm{~Hz}, 1 \mathrm{H}), 8.25(\mathrm{~s}, 1 \mathrm{H}), 7.90(\mathrm{~d}, J=$ $3.5 \mathrm{~Hz}, 1 \mathrm{H}), 7.60-7.51$ (m, 4H), 7.49-7.44 (m, 3H), 7.06 (dd, $J=8.7,2.0 \mathrm{~Hz}, 1 \mathrm{H}), 6.97-6.92(\mathrm{~m}, 2 \mathrm{H}), 5.91(\mathrm{~d}, J=2.0 \mathrm{~Hz}$, 1H), 3.92 (s, 3H); EI-MS: $453[\mathrm{M}+1]^{+}$.

3-(5-Bromo-1-methyl-1H-indol-3-yl)-1-phenyl-4-(1Hpyrrolo[3,2-c]pyridin-1-yl)-1H-pyrrole-2,5-dione (3c): Orange solid, m.p. $103-105^{\circ} \mathrm{C}$; ${ }^{1} \mathrm{H}$ NMR $(500 \mathrm{MHz}, \mathrm{DMSO}-$ $\left.d_{6}\right) \delta 8.32(\mathrm{dd}, J=4.6,1.3 \mathrm{~Hz}, 1 \mathrm{H}), 8.23(\mathrm{~s}, 1 \mathrm{H}), 7.89(\mathrm{~d}, J=$ $3.5 \mathrm{~Hz}, 1 \mathrm{H}), 7.61-7.50(\mathrm{~m}, 4 \mathrm{H}), 7.50-7.39$ (m, 3H), 7.17 (dd, $J=8.6,1.6 \mathrm{~Hz}, 1 \mathrm{H}), 6.98-6.91(\mathrm{~m}, 2 \mathrm{H}), 6.06(\mathrm{~d}, J=1.6 \mathrm{~Hz}$, 1H), 3.92 (s, 3H); EI-MS: $498[\mathrm{M}+1]^{+}$.

3-(5-Methoxy-1-methyl-1H-indol-3-yl)-1-phenyl-4(1H-pyrrolo[3,2-c]pyridin-1-yl)-1H-pyrrole-2,5-dione (3d): Orange solid, m.p. $123-125{ }^{\circ} \mathrm{C}$; ${ }^{1} \mathrm{H}$ NMR $(500 \mathrm{MHz}$, DMSO$\left.d_{6}\right) \delta 8.35(\mathrm{dd}, J=4.6,1.3 \mathrm{~Hz}, 1 \mathrm{H}), 8.21(\mathrm{~s}, 1 \mathrm{H}), 7.86(\mathrm{~d}, J=$ $3.5 \mathrm{~Hz}, 1 \mathrm{H}), 7.68-7.63(\mathrm{~m}, 1 \mathrm{H}), 7.59-7.51(\mathrm{~m}, 4 \mathrm{H}), 7.47-7.43$ $(\mathrm{m}, 1 \mathrm{H}), 7.34(\mathrm{~d}, J=8.9 \mathrm{~Hz}, 1 \mathrm{H}), 7.03-7.00(\mathrm{~m}, 1 \mathrm{H}), 6.88(\mathrm{~d}$, $J=3.5 \mathrm{~Hz}, 1 \mathrm{H}), 6.64(\mathrm{dd}, J=8.8,2.4 \mathrm{~Hz}, 1 \mathrm{H}), 5.48(\mathrm{~d}, J=2.4$ $\mathrm{Hz}, 1 \mathrm{H}), 3.91$ (s, 3H), 2.97 (s, 3H); EI-MS: $499[\mathrm{M}+1]^{+}$.

3-(1,7-Dimethyl-1H-indol-3-yl)-1-phenyl-4-(1Hpyrrolo[3,2-c]pyridin-1-yl)-1 $\boldsymbol{H}$-pyrrole-2,5-dione (3e): Red solid, m.p. $134-135{ }^{\circ} \mathrm{C} ;{ }^{1} \mathrm{H}$ NMR $\left(500 \mathrm{MHz}\right.$, DMSO- $\left.d_{6}\right) \delta 8.31$ $(\mathrm{dd}, J=4.6,1.3 \mathrm{~Hz}, 1 \mathrm{H}), 8.11(\mathrm{~s}, 1 \mathrm{H}), 7.84(\mathrm{~d}, J=3.6 \mathrm{~Hz}, 1 \mathrm{H})$, 7.59-7.51 (m, 5H), 7.47-7.43 (m, 1H), 6.98-6.94 (m, 1H), 6.89-6.87 $(\mathrm{m}, 1 \mathrm{H}), 6.72(\mathrm{~d}, J=7.1 \mathrm{~Hz}, 1 \mathrm{H}), 6.40(\mathrm{t}, J=7.9 \mathrm{~Hz}, 1 \mathrm{H}), 5.88$ $(\mathrm{d}, J=8.0 \mathrm{~Hz}, 1 \mathrm{H}), 4.19$ (s, 3H), 2.68 (s, 3H); EI-MS: $433[\mathrm{M}+1]^{+}$.

3-(1-Methyl-1H-indol-3-yl)-1-phenyl-4-(1H-pyrrolo[3,2-b]pyridin-1-yl)-1H-pyrrole-2,5-dione (3f): Orange solid, m.p. > $250{ }^{\circ} \mathrm{C} ;{ }^{1} \mathrm{H}$ NMR (500 MHz, DMSO- $\left.d_{6}\right) \delta 8.30$ $(\mathrm{dd}, J=4.5,1.1 \mathrm{~Hz}, 1 \mathrm{H}), 8.24(\mathrm{~s}, 1 \mathrm{H}), 7.89$ (d, $J=3.5 \mathrm{~Hz}$,
1H), 7.61-7.52 (m, 4H), $7.50(\mathrm{~d}, J=8.3 \mathrm{~Hz}, 1 \mathrm{H}), 7.48-7.41(\mathrm{~m}$, 2H), 7.04 (t, $J=7.6 \mathrm{~Hz}, 1 \mathrm{H}), 6.97-6.87(\mathrm{~m}, 2 \mathrm{H}), 6.57$ (t, $J=$ $7.5 \mathrm{~Hz}, 1 \mathrm{H}), 6.00(\mathrm{~d}, J=8.1 \mathrm{~Hz}, 1 \mathrm{H}), 3.93$ (s, 3H); EI-MS: $419[\mathrm{M}+1]^{+}$.

3-(1-Methyl-1H-indol-3-yl)-1-phenyl-4-(1H-pyrrolo[2,3-c]pyridin-1-yl)-1H-pyrrole-2,5-dione (3g): Orange solid, m.p. $162-163{ }^{\circ} \mathrm{C} ;{ }^{1} \mathrm{H}$ NMR $\left(500 \mathrm{MHz}, \mathrm{DMSO}-d_{6}\right) \delta$ $8.42(\mathrm{~s}, 1 \mathrm{H}), 8.26(\mathrm{~s}, 1 \mathrm{H}), 8.07$ (d, $J=5.4 \mathrm{~Hz}, 1 \mathrm{H}), 7.85$ (d, $J=3.3 \mathrm{~Hz}, 1 \mathrm{H}), 7.61-7.52(\mathrm{~m}, 5 \mathrm{H}), 7.49-7.42(\mathrm{~m}, 2 \mathrm{H}), 7.04$ $(\mathrm{td}, J=7.6,0.6 \mathrm{~Hz}, 1 \mathrm{H}), 6.88(\mathrm{~d}, J=3.1 \mathrm{~Hz}, 1 \mathrm{H}), 6.56(\mathrm{t}, J=$ $7.5 \mathrm{~Hz}, 1 \mathrm{H}), 5.97(\mathrm{~d}, J=8.1 \mathrm{~Hz}, 1 \mathrm{H}), 3.94$ (s, 3H); EI-MS: $419[\mathrm{M}+1]^{+}$.

3-(1-Methyl-1H-indol-3-yl)-1-phenyl-4-(1H-pyrrolo[2,3-b]pyridin-1-yl)-1H-pyrrole-2,5-dione (3h): Orange solid, m.p. 199-200 ${ }^{\circ} \mathrm{C} ;{ }^{1} \mathrm{H}$ NMR $\left(500 \mathrm{MHz}, \mathrm{DMSO}-d_{6}\right) \delta$ 8.30 (s, 1H), 8.05 (dd, $J=7.8,1.5 \mathrm{~Hz}, 1 \mathrm{H}), 8.00$ (dd, $J=4.7$, $1.5 \mathrm{~Hz}, 1 \mathrm{H}), 7.74(\mathrm{~d}, J=3.7 \mathrm{~Hz}, 1 \mathrm{H}), 7.59-7.50(\mathrm{~m}, 4 \mathrm{H})$, 7.48-7.44 (m, 1H), $7.42(\mathrm{~d}, J=8.2 \mathrm{~Hz}, 1 \mathrm{H}), 7.08-7.02(\mathrm{~m}$, $1 \mathrm{H}), 7.02$ (t, $J=7.8 \mathrm{~Hz}, 1 \mathrm{H}), 6.83(\mathrm{~d}, J=3.7 \mathrm{~Hz}, 1 \mathrm{H}), 6.52$ $(\mathrm{t}, J=7.7 \mathrm{~Hz}, 1 \mathrm{H}), 5.90(\mathrm{~d}, J=8.1 \mathrm{~Hz}, 1 \mathrm{H}), 3.91(\mathrm{~s}, 3 \mathrm{H})$; EIMS: $419[\mathrm{M}+1]^{+}$.

3-(1H-Benzo[d]imidazol-1-yl)-4-(1-methyl-1H-indol-3yl)-1-phenyl-1H-pyrrole-2,5-dione (3i): Red solid, m.p. 200$201{ }^{\circ} \mathrm{C}$; ${ }^{1} \mathrm{H}$ NMR $\left(500 \mathrm{MHz}, \mathrm{DMSO}-d_{6}\right) \delta 8.45(\mathrm{~s}, 1 \mathrm{H}), 8.29$ (s, 1H), $7.74(\mathrm{~d}, J=7.9 \mathrm{~Hz}, 1 \mathrm{H}), 7.63-7.51(\mathrm{~m}, 4 \mathrm{H}), 7.51-7.43$ (m, 2H), 7.30-7.16 (m, 2H), 7.14-7.03 (m, 2H), $6.61(\mathrm{t}, J=$ $7.4 \mathrm{~Hz}, 1 \mathrm{H}), 6.08$ (d, $J=7.9 \mathrm{~Hz}, 1 \mathrm{H}), 3.94$ (s, 3H); EI-MS: $419[\mathrm{M}+1]^{+}$.

3-(1-Methyl-1H-indol-3-yl)-1-phenyl-4-(1H-1,2,4triazol-1-yl)-1H-pyrrole-2,5-dione (3j): Orange solid, m.p. 174-176 ${ }^{\circ} \mathrm{C}$; ${ }^{1} \mathrm{H}$ NMR $\left(500 \mathrm{MHz}, \mathrm{DMSO}-d_{6}\right) \delta 8.93(\mathrm{~s}, 1 \mathrm{H})$, $8.28(\mathrm{~s}, 2 \mathrm{H}), 7.60-7.53(\mathrm{~m}, 3 \mathrm{H}), 7.53-7.44(\mathrm{~m}, 3 \mathrm{H}), 7.23(\mathrm{t}$, $J=7.5 \mathrm{~Hz}, 1 \mathrm{H}), 6.92(\mathrm{t}, J=7.5 \mathrm{~Hz}, 1 \mathrm{H}), 6.22(\mathrm{~d}, J=8.0 \mathrm{~Hz}$, 1H), 3.95 (s, 3H); EI-MS: $370[\mathrm{M}+1]^{+}$.

General procedure for the synthesis of $4 \mathrm{a}-4 \mathrm{j}$ : A mixture of compound $\mathbf{3}(0.1 \mathrm{mmol})$ and ammonium acetate $(1.39 \mathrm{~g}$, $18 \mathrm{mmol}$ ) was heated by microwave irradiation at $140{ }^{\circ} \mathrm{C}$ for $20 \mathrm{~min}$. After cooling, the resulted reaction mixture was poured into water $(50 \mathrm{~mL})$, adjusted to weak alkalinity with $\mathrm{Na}_{2} \mathrm{CO}_{3}$ and extracted with ethyl acetate $(3 \times 50 \mathrm{~mL})$. The organic layer was washed with brine $(3 \times 150 \mathrm{~mL})$, dried over $\mathrm{Na}_{2} \mathrm{SO}_{4}$ and concentrated in vacuo. The residue was purified by flash column chromatography on silica gel using ethyl acetate/petroleum ether $(2: 1, \mathrm{v} / \mathrm{v})$ as eluent to afford compound $\mathbf{4 a - 4 i}$.

3-(1-Methyl-1H-indol-3-yl)-4-(1H-pyrrolo[3,2c]pyridin-1-yl)-1H-pyrrole-2,5-dione (4a): Orange solid, m.p. $>250{ }^{\circ} \mathrm{C} ;{ }^{1} \mathrm{H}$ NMR $\left(500 \mathrm{MHz}, \mathrm{DMSO}-d_{6}\right) \delta 11.30$ (brs, $1 \mathrm{H}), 8.27(\mathrm{dd}, J=4.6,1.2 \mathrm{~Hz}, 1 \mathrm{H}), 8.17(\mathrm{~s}, 1 \mathrm{H}), 7.84(\mathrm{~d}, J=$ $3.5 \mathrm{~Hz}, 1 \mathrm{H}), 7.41(\mathrm{~d}, J=8.2 \mathrm{~Hz}, 1 \mathrm{H}), 7.35(\mathrm{~d}, J=8.3 \mathrm{~Hz}, 1 \mathrm{H})$, $7.01(\mathrm{t}, J=7.6 \mathrm{~Hz}, 1 \mathrm{H}), 6.90-6.85(\mathrm{~m}, 2 \mathrm{H}), 6.52(\mathrm{t}, J=7.5 \mathrm{~Hz}$, $1 \mathrm{H}), 5.91(\mathrm{~d}, J=8.0 \mathrm{~Hz}, 1 \mathrm{H}), 3.90(\mathrm{~s}, 3 \mathrm{H})$; EI-MS: $343[\mathrm{M}+1]^{+}$.

3-(5-Chloro-1-methyl-1H-indol-3-yl)-4-(1H-pyrrolo[3,2-c]pyridin-1-yl)-1H-pyrrole-2,5-dione (4b): Orange solid, m.p. > $250{ }^{\circ} \mathrm{C} ;{ }^{1} \mathrm{H}$ NMR (500 MHz, DMSO- $\left.d_{6}\right) \delta 11.33$ (brs, 1H), 8.29 (dd, $J=4.6,1.3 \mathrm{~Hz}, 1 \mathrm{H}), 8.19$ (s, 1H), 7.86 (d, $J=3.5 \mathrm{~Hz}, 1 \mathrm{H}), 7.43(\mathrm{~d}, J=8.7 \mathrm{~Hz}, 1 \mathrm{H}), 7.32(\mathrm{~d}, J=8.3 \mathrm{~Hz}$, $1 \mathrm{H}), 7.02(\mathrm{dd}, J=8.7,2.0 \mathrm{~Hz}, 1 \mathrm{H}), 6.92(\mathrm{~d}, J=3.5 \mathrm{~Hz}, 1 \mathrm{H})$, 6.90-6.86 (m, 1H), $5.80(\mathrm{~d}, J=20 \mathrm{~Hz}, 1 \mathrm{H}), 3.91$ (s, 3H); EI-MS: $377[\mathrm{M}+1]^{+}$. 
3-(5-Bromo-1-methyl-1H-indol-3-yl)-4-(1H-pyrrolo[3,2-c]pyridin-1-yl)-1H-pyrrole-2,5-dione (4c): Yellow solid, m.p. $>250{ }^{\circ} \mathrm{C} ;{ }^{1} \mathrm{H}$ NMR $\left(500 \mathrm{MHz}, \mathrm{DMSO}-d_{6}\right) \delta 11.33$ (brs, $1 \mathrm{H}), 8.29(\mathrm{dd}, J=4.6,1.3 \mathrm{~Hz}, 1 \mathrm{H}), 8.17(\mathrm{~s}, 1 \mathrm{H}), 7.85$ (d, $J=$ $3.5 \mathrm{~Hz}, 1 \mathrm{H}), 7.38(\mathrm{~d}, J=8.7 \mathrm{~Hz}, 1 \mathrm{H}), 7.33(\mathrm{~d}, J=8.3 \mathrm{~Hz}, 1 \mathrm{H})$, $7.13(\mathrm{dd}, J=8.7,1.8 \mathrm{~Hz}, 1 \mathrm{H}), 6.92(\mathrm{~d}, J=3.2 \mathrm{~Hz}, 1 \mathrm{H}), 6.90$ $6.86(\mathrm{~m}, 1 \mathrm{H}), 5.94(\mathrm{~d}, J=1.8 \mathrm{~Hz}, 1 \mathrm{H}), 3.89(\mathrm{~s}, 3 \mathrm{H})$; EI-MS:322 $[\mathrm{M}+1]^{+}$.

3-(5-Methoxy-1-methyl-1H-indol-3-yl)-4-(1H-pyrrolo[3,2-c]pyridin-1-yl)-1H-pyrrole-2,5-dione (4d): Orange solid, m.p. $>250{ }^{\circ} \mathrm{C} ;{ }^{1} \mathrm{H}$ NMR (500 MHz, DMSO- $\left.d_{6}\right) \delta 11.28$ (s, 1H), 8.27 (dd, $J=4.5,1.0 \mathrm{~Hz}, 1 \mathrm{H}), 8.03$ (s, 1H), 7.80 (d, $J$ $=3.4 \mathrm{~Hz}, 1 \mathrm{H}), 7.36(\mathrm{~d}, J=8.3 \mathrm{~Hz}, 1 \mathrm{H}), 6.93-6.88(\mathrm{~m}, 1 \mathrm{H})$, $6.84(\mathrm{~d}, J=3.3 \mathrm{~Hz}, 1 \mathrm{H}), 6.69(\mathrm{~d}, J=7.2 \mathrm{~Hz}, 1 \mathrm{H}), 6.35(\mathrm{t}, J=$ $7.6 \mathrm{~Hz}, 1 \mathrm{H}), 5.79(\mathrm{~d}, J=8.1 \mathrm{~Hz}, 1 \mathrm{H}), 4.16(\mathrm{~s}, 3 \mathrm{H}), 2.66(\mathrm{~s}$, 3H); EI-MS: $357[\mathrm{M}+1]^{+}$.

3-(1,7-Dimethyl-1H-indol-3-yl)-4-(1H-pyrrolo[3,2c]pyridin-1-yl)-1H-pyrrole-2,5-dione (4e): Orange solid, m.p. $>250{ }^{\circ} \mathrm{C} ;{ }^{1} \mathrm{H}$ NMR $(500 \mathrm{MHz}$, DMSO-d 6 ) $\delta 11.28$ $(\mathrm{s}, 1 \mathrm{H}), 8.32(\mathrm{dd}, J=4.6,1.3 \mathrm{~Hz}, 1 \mathrm{H}), 8.15(\mathrm{~s}, 1 \mathrm{H}), 7.82$ (d, $J$ $=3.4 \mathrm{~Hz}, 1 \mathrm{H}), 7.48(\mathrm{~d}, J=8.3 \mathrm{~Hz}, 1 \mathrm{H}), 7.30(\mathrm{~d}, J=8.9 \mathrm{~Hz}$, $1 \mathrm{H}), 6.98-6.94(\mathrm{~m}, 1 \mathrm{H}), 6.84(\mathrm{~d}, J=3.8 \mathrm{~Hz}, 1 \mathrm{H}), 6.60$ (dd, $J=8.9,2.4 \mathrm{~Hz}, 1 \mathrm{H}), 5.40(\mathrm{~d}, J=2.4 \mathrm{~Hz}, 1 \mathrm{H}), 3.88(\mathrm{~s}, 3 \mathrm{H})$, 2.95 (s, 3H); EI-MS: $373[\mathrm{M}+1]^{+}$

3-(1-Methyl-1 $H$-indol-3-yl)-4-(1H-pyrrolo[3,2-b]pyridin1-yl)-1 $\boldsymbol{H}$-pyrrole-2,5-dione (4f): Orange solid, m.p. $>250{ }^{\circ} \mathrm{C}$; ${ }^{1} \mathrm{H}$ NMR $\left(500 \mathrm{MHz}\right.$, DMSO- $\left.d_{6}\right) \delta 11.30$ (brs, $1 \mathrm{H}$ ), 8.27 (d, $J=$ $4.1 \mathrm{~Hz}, 1 \mathrm{H}), 8.17(\mathrm{~s}, 1 \mathrm{H}), 7.84(\mathrm{~d}, J=3.3 \mathrm{~Hz}, 1 \mathrm{H}), 7.41(\mathrm{~d}$, $J=8.2 \mathrm{~Hz}, 1 \mathrm{H}), 7.35(\mathrm{~d}, J=8.3 \mathrm{~Hz}, 1 \mathrm{H}), 7.01(\mathrm{t}, J=7.6 \mathrm{~Hz}$, $1 \mathrm{H}), 6.91-6.85(\mathrm{~m}, 2 \mathrm{H}), 6.52(\mathrm{t}, J=7.6 \mathrm{~Hz}, 1 \mathrm{H}), 5.91(\mathrm{~d}, J=$ $8.0 \mathrm{~Hz}, 1 \mathrm{H}), 3.90$ (s, 3H); EI-MS: $343[\mathrm{M}+1]^{+}$.

3-(1-Methyl-1H-indol-3-yl)-4-(1H-pyrrolo[2,3-c]pyridin1-yl)-1H-pyrrole-2,5-dione (4g): Orange solid, m.p. $>250{ }^{\circ} \mathrm{C}$;
${ }^{1} \mathrm{H}$ NMR $\left(500 \mathrm{MHz}, \mathrm{DMSO}-d_{6}\right) \delta 11.32$ (brs, 1H), $8.27(\mathrm{~s}$, $1 \mathrm{H}), 8.19(\mathrm{~s}, 1 \mathrm{H}), 8.03(\mathrm{~d}, J=5.3 \mathrm{~Hz}, 1 \mathrm{H}), 7.81(\mathrm{~d}, J=3.2 \mathrm{~Hz}$, $1 \mathrm{H}), 7.55(\mathrm{~d}, J=5.2 \mathrm{~Hz}, 1 \mathrm{H}), 7.42(\mathrm{~d}, J=8.2 \mathrm{~Hz}, 1 \mathrm{H}), 7.01(\mathrm{t}$, $J=7.6 \mathrm{~Hz}, 1 \mathrm{H}), 6.84(\mathrm{~d}, J=3.1 \mathrm{~Hz}, 1 \mathrm{H}), 6.51(\mathrm{t}, J=7.6 \mathrm{~Hz}$, $1 \mathrm{H}), 5.88(\mathrm{~d}, J=8.1 \mathrm{~Hz}, 1 \mathrm{H}), 3.92(\mathrm{~s}, 3 \mathrm{H})$; EI-MS: $343[\mathrm{M}+1]^{+}$.

3-(1-Methyl-1H-indol-3-yl)-4-(1H-pyrrolo[2,3-b]pyridin-1-yl)-1H-pyrrole-2,5-dione (4h): Orange solid, m.p. $>250{ }^{\circ} \mathrm{C} ;{ }^{1} \mathrm{H}$ NMR $\left(500 \mathrm{MHz}\right.$, DMSO- $\left.d_{6}\right) \delta 11.28$ (brs, $\left.1 \mathrm{H}\right)$, $8.23(\mathrm{~s}, 1 \mathrm{H}), 8.03$ (dd, $J=7.8,1.5 \mathrm{~Hz}, 1 \mathrm{H}), 7.98$ (dd, $J=4.7$, $1.4 \mathrm{~Hz}, 1 \mathrm{H}), 7.67(\mathrm{~d}, J=3.6 \mathrm{~Hz}, 1 \mathrm{H}), 7.40(\mathrm{~d}, J=8.2 \mathrm{~Hz}, 1 \mathrm{H})$, 7.08-7.04 (m, 1H), $7.00(\mathrm{t}, J=7.4 \mathrm{~Hz}, 1 \mathrm{H}), 6.77(\mathrm{~d}, J=3.6$ $\mathrm{Hz}, 1 \mathrm{H}), 6.48$ (t, $J=7.5 \mathrm{~Hz}, 1 \mathrm{H}), 5.83(\mathrm{~d}, J=8.1 \mathrm{~Hz}, 1 \mathrm{H})$, 3.88 (s, 3H); EI-MS: $343[\mathrm{M}+1]^{+}$.

3-(1H-Benzo[d]imidazol-1-yl)-4-(1-methyl-1H-indol-3yl)-1H-pyrrole-2,5-dione (4i): Brown solid, m.p. > $250{ }^{\circ} \mathrm{C}$; ${ }^{1} \mathrm{H}$ NMR (500 MHz, DMSO-d 6 ) $\delta 11.41$ (brs, 1H), 8.42 (s, $1 \mathrm{H}), 8.22(\mathrm{~s}, 1 \mathrm{H}), 7.71(\mathrm{~d}, J=8.0 \mathrm{~Hz}, 1 \mathrm{H}), 7.44(\mathrm{~d}, J=8.2 \mathrm{~Hz}$, $1 \mathrm{H}), 7.17(\mathrm{~m}, J=7.20-7.15 \mathrm{~Hz}, 1 \mathrm{H}), 7.10(\mathrm{~d}, J=8.0 \mathrm{~Hz}, 1 \mathrm{H})$, 7.06-7.01 (m, 2H), $6.56(\mathrm{t}, J=7.5 \mathrm{~Hz}, 1 \mathrm{H}), 5.99(\mathrm{~d}, J=8.0$ Hz, 1H), 3.91 (s, 3H); EI-MS: $343.2[\mathrm{M}+1]^{+}$.

3-(1-methyl-1H-indol-3-yl)-4-(1H-1,2,4-triazol-1-yl)$1 \boldsymbol{H}$-pyrrole-2,5-dione (4j): Dark red solid, m.p. 232-234 ${ }^{\circ} \mathrm{C}$; ${ }^{1} \mathrm{H}$ NMR (500 MHz, DMSO- $\left.d_{6}\right) \delta 10.22$ (brs, 1H), 7.64 (d, $J=$ $8.0 \mathrm{~Hz}, 1 \mathrm{H}), 7.46-7.42(\mathrm{~m}, 2 \mathrm{H}), 7.19-7.16(\mathrm{~m}, 1 \mathrm{H}), 7.05(\mathrm{t}$, $J=7.3 \mathrm{~Hz}, 1 \mathrm{H}), 6.69$ (brs, 2H), 3.81 (s, 3H); EI-MS: 294.1 $[\mathrm{M}+1]^{+}$.

\section{RESULTS AND DISCUSSION}

To get a good yield for the synthesis of $\mathbf{3}$, the condensation of 1a with 2a to afford $\mathbf{3 a}$ was selected as a model reaction to survey the reaction condition. In a mild reaction environment, different solvents were first investigated (Table-1, Entry 1-5, 7-9). Polar solvents such as DMF or MeCN afforded a

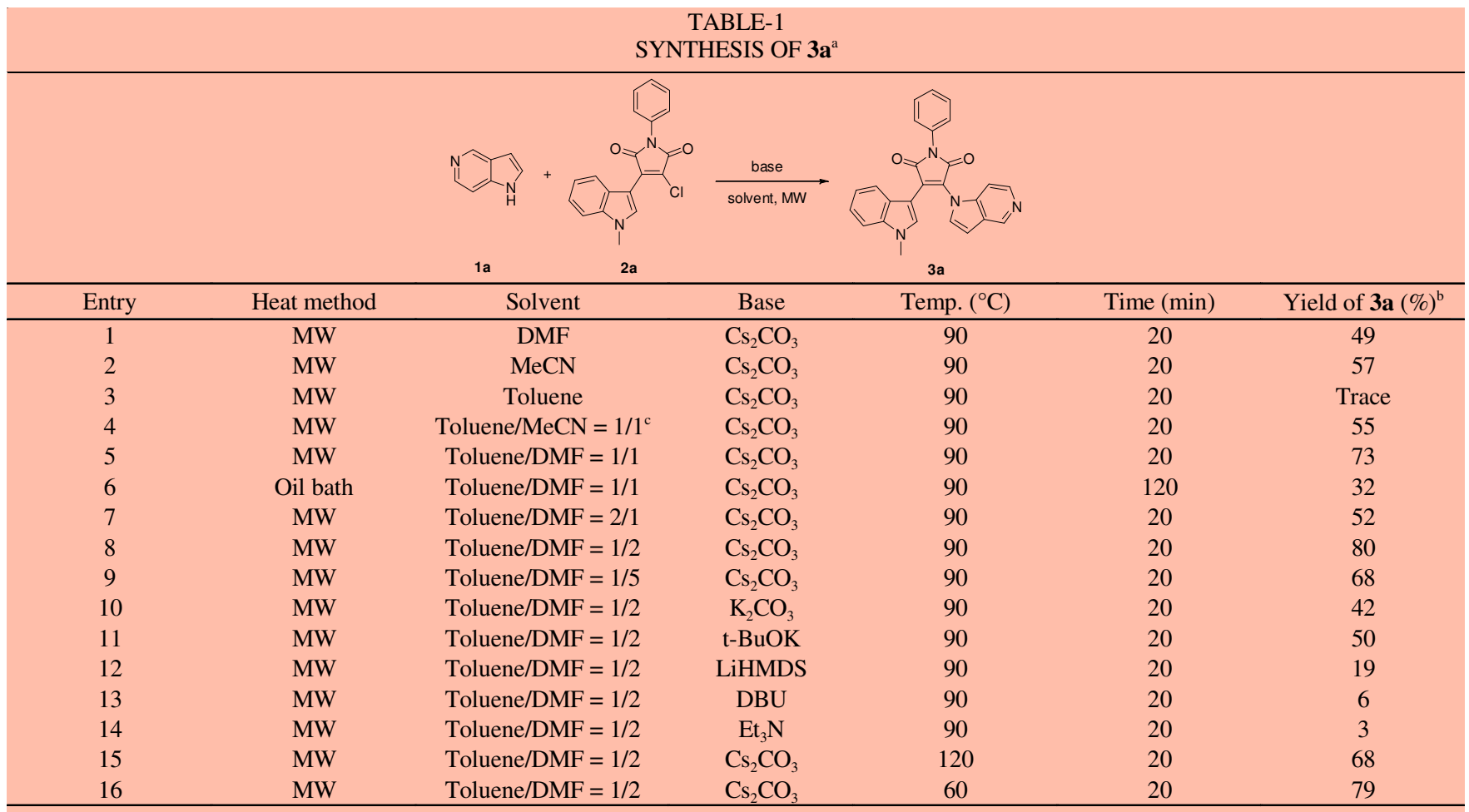

${ }^{\mathrm{a}}$ Reactions were run using $0.12 \mathrm{mmol}$ of $\mathbf{1 a}, 0.1 \mathrm{mmol}$ of $\mathbf{2 a}$, and $0.2 \mathrm{mmol}$ of base in $2.0 \mathrm{~mL}$ of solvent. ${ }^{\mathrm{b}}$ Isolated yields. ${ }^{\mathrm{c}}$ The solvent was mixed in volume ratio, and the total volume was $2 \mathrm{~mL}$. 
moderate yield $(50 \%)$. However, only trace of $\mathbf{3 a}$ could be found when nonpolar toluene was used as the solvent. The yield was improved by using a mixed solution of DMF and toluene and the best yield $(80 \%)$ was obtained when the volume of DMF was 2 times of toluene. We next shifted our focus to other bases such as $\mathrm{K}_{2} \mathrm{CO}_{3}, t$-BuOK, LiHMDS, DBU and $\mathrm{Et}_{3} \mathrm{~N}$ (Table-1, Entry 10-14). However, no better yields were obtained. Finally, different reaction temperatures were studied. When the reaction temperature was increased to $120^{\circ} \mathrm{C}$ or decreased to $60{ }^{\circ} \mathrm{C}$, it was not helpful to increase the yield (Table-1, Entry 15-16). As compared to using a conventional reaction condition (entry 6), the microwave irradiation displayed extreme efficiency, which promoted the reaction to completion in $20 \mathrm{~min}$ and also in high yield (entry 5 ). Therefore, optimized conditions involved microwave assisted condensation of $\mathbf{1 a}$ (1.2 equiv) with $\mathbf{2 b}\left(1.0\right.$ equiv) at $90^{\circ} \mathrm{C}$ in a mixed solution (DMF: toluene $=2: 1 \mathrm{v}: \mathrm{v}$ ), using $\mathrm{Cs}_{2} \mathrm{CO}_{3}$ (2 equiv) as the base, which gave $3 \mathbf{a}$ in $80 \%$ yield.

To investigate the scope of the microwave assisted condensation reaction, several $\mathrm{N}$-containing heterocycles 1a-1f were selected to react with different substituted monochloromaleimides $\mathbf{2}$ and the results were listed in Table-2.

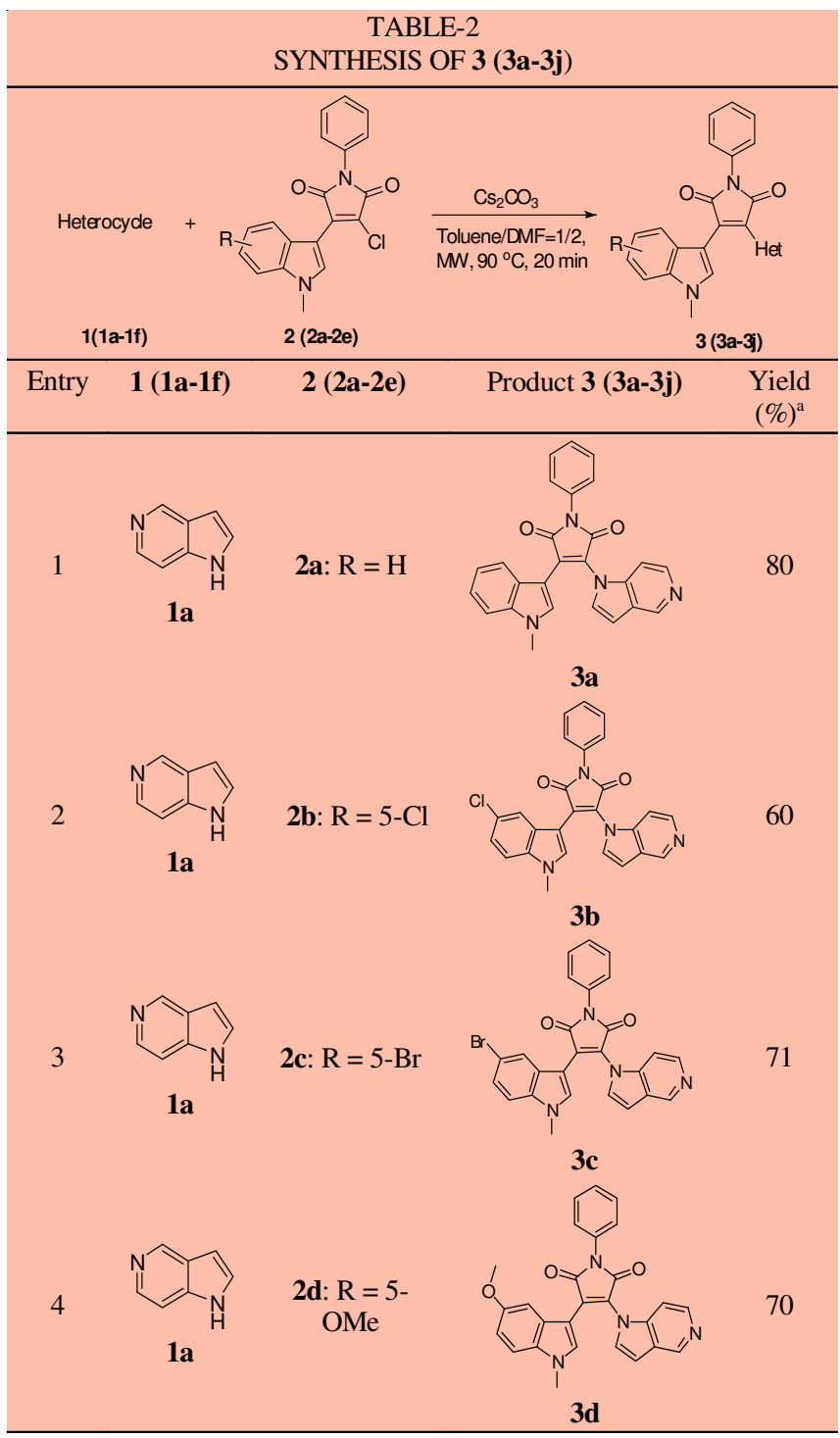

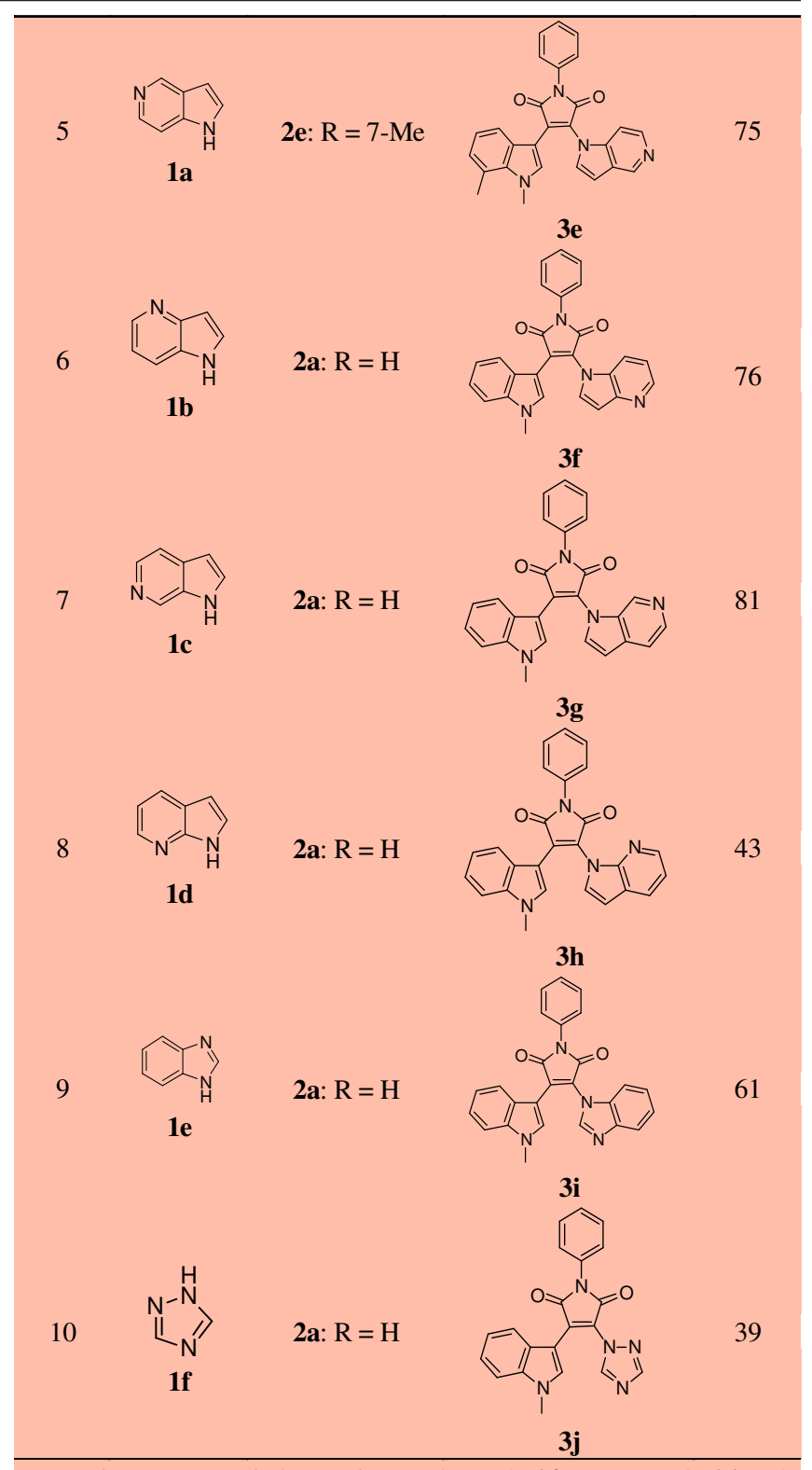

${ }^{a}$ Reactions were carried out using $0.15 \mathrm{mmol}$ of $\mathbf{2}, 0.18 \mathrm{mmol}$ of $\mathbf{1}$ and $0.3 \mathrm{mmol}$ of cesium carbonate in mixed solvent of DMF $(2 \mathrm{~mL})$ and Toluene $(1.0 \mathrm{~mL})$; isolated yields

It is observed that the azaindoles, benzoimidazole and 1,3,4-trizazole are all able to react with different substituted monochloromaleimide to get the target products and the yields were affected to some extent by the structure of heterocycle and monochloromaleimide. When 4-azaindole, 5-azaindole or 6-azaindole was employed in the microwave assisted condensation, the yield was relatively high (Table-2, Entry 1, 6, 7). However, using 7-azaindole or 1,3,4-triazole in the reaction was unfavorable for the yield (Table 2, Entry 8, 10). All the synthesized $\mathbf{3 a - 3}$ j can be converted to potential GSK-3 inhibitors $\mathbf{4} \mathbf{a}-\mathbf{4 j}$ easily by a microwave assisted ammonolysis in yield 55-98 \% (Table-3) and the microwave irradiation was also superior to the conventional reaction condition.

\section{Conclusion}

We have reported a convenient, simple and efficient synthesis of some new monoindolylmaleimides via microwave assisted condensation of substituted monochloro-maleimides 
TABLE-3

SYNTHESIS OF $4(\mathbf{4} \mathbf{a}-\mathbf{4 j})$

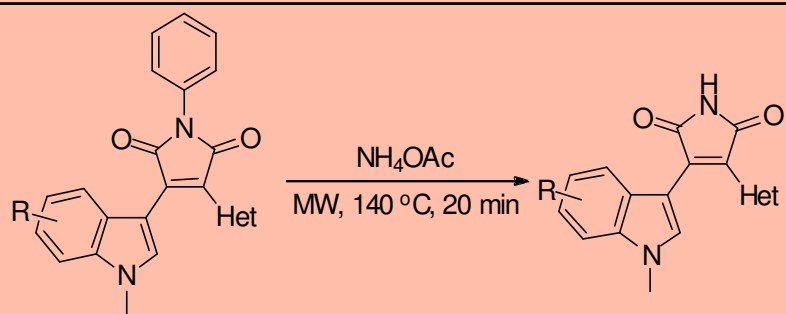

$3(3 a 3 j) \quad 4(4 a-4 j)$

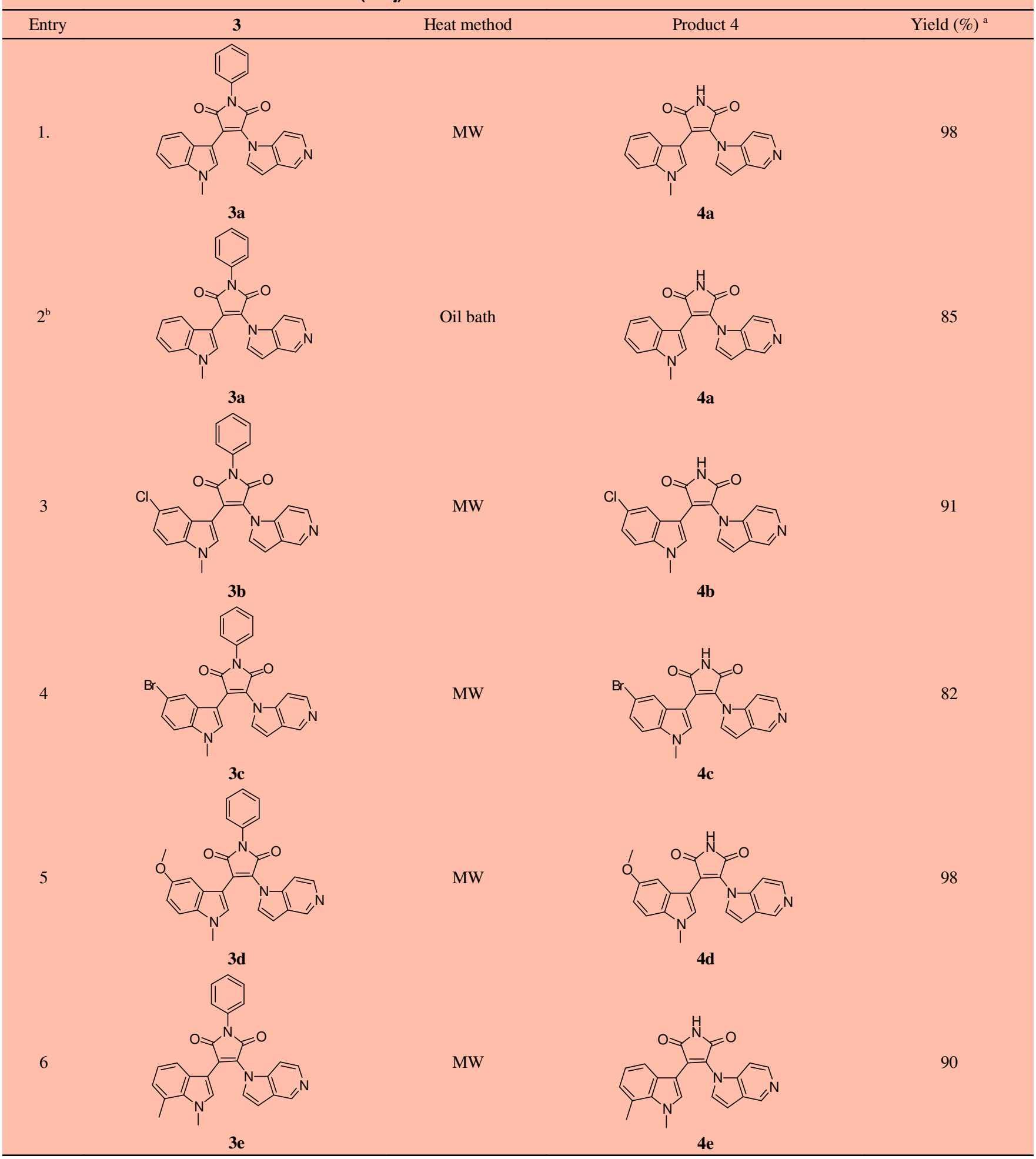


7<smiles>Cn1cc(C2=C(n3ccc4ncccc43)C(=O)N(c3ccccc3)C2=O)c2ccccc21</smiles>

8<smiles>Cn1cc(C2=C(n3ccc4ccncc43)C(=O)N(c3ccccc3)C2=O)c2ccccc21</smiles>

$3 g$<smiles>Cn1cc(C2=C(n3ccc4cccnc43)C(=O)N(c3ccccc3)C2=O)c2ccccc21</smiles>

3h<smiles>Cn1cc(C2=C(n3cnc4ccccc43)C(=O)N(c3ccccc3)C2=O)c2ccccc21</smiles>

$3 \mathbf{i}$<smiles>Cn1cc(C2=C(n3cncn3)C(=O)N(c3ccccc3)C2=O)c2ccccc21</smiles>

3j
MW<smiles>Cn1cc(C2=C(n3ccc4ncccc43)C(=O)NC2=O)c2ccccc21</smiles>

$4 f$<smiles>Cn1cc(C2=C(n3ccc4ccncc43)C(=O)NC2=O)c2ccccc21</smiles>

$4 g$<smiles>Cn1cc(C2=C(n3ccc4cccnc43)C(=O)NC2=O)c2ccccc21</smiles>

4h<smiles>Cn1cc(C2=C(n3cnc4ccccc43)C(=O)NC2=O)c2ccccc21</smiles>

$4 i$

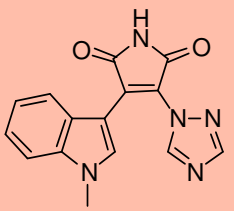

$4 j$
99

${ }^{a}$ Reactions were carried out using $0.1 \mathrm{mmol}$ of $\mathbf{3}$ and $240 \mathrm{mmol}$ ammonium acetate for $20 \mathrm{~min}$; isolated yields. ${ }^{\mathrm{b}} \mathrm{Reaction}$ was carried out for $4 \mathrm{~h}$

with N-containing heterocycles followed by ammonolysis. Further studies of the scope of the reaction and the pharmacological activity of the new monoindolylmaleimides are currently in progress.

\section{REFERENCES}

1. T.A. Engler, J.R. Henry, S. Malhotra, B. Cunningham, K. Furness, J. Brozinick, T.P. Burkholder, M.P. Clay, J. Clayton, C. Diefenbacher, E. Hawkins, P.W. Iversen, Y. Li, T.D. Lindstrom, A.L. Marquart, J. McLean, D. Mendel, E. Misener, D. Briere, J.C. O'Toole, W.J. Porter, S. Queener, J.K. Reel, R.A. Owens, R.A. Brier, T.E. Eessalu, J.R. Wagner, R.M. Campbell and R. Vaughn, J. Med. Chem., 47, 3934 (2004).

2. I.N. Gaisina, F. Gallier, A.V. Ougolkov, K.H. Kim, T. Kurome, S. Guo, D. Holzle, D.N. Luchini, S.Y. Blond, D.D. Billadeau and A.P. Kozikowski, J. Med. Chem., 52, 1853 (2009).
3. H.C.Zhang, C.K. Derian, D.F. McComsey, K.B. White, H. Ye, L.R. Hecker, J. Li, M.F. Addo, D. Croll, A.J. Eckardt, C.E. Smith, Q. Li, W.M. Cheung, B.R. Conway, S. Emanuel, K.T. Demarest, P. Andrade-Gordon, B.P. Damiano and B.E. Maryanoff, J. Med. Chem., 48, 1725 (2005).

4. L. Kim and A.R. Kimmel, Curr. Opin. Genet. Dev., 10, 508 (2000).

5. A.S. Wagman and J.M. Nuss, Curr. Pharm. Des., 7, 417 (2001).

6. P. Cohen, Eur. J. Biochem., 268, 5001 (2001).

7. C. Sasaki, T. Hayashi, W.R. Zhang, H. Warita, Y. Manabe, K. Sakai and K. Abe, Neurol. Res., 23, 588 (2001).

8. A. Castro and A. Martinez, Exp. Opin. Ther. Pat., 10, 1519 (2000).

9. Q. Ye, M. Li, Y. Zhou, T. Pang, L. Xu, J. Cao, L. Han, Y. Li, W. Wang, J. Gao and J. Li, Molecules, 18, 5498 (2013).

10. Q. Ye, M. Li, Y.-B. Zhou, J.-Y. Cao, L. Xu, Y.-J. Li, L. Han, J.-R. Gao, Y.-Z. Hu and J. Li, Arch. Pharm. (Weinheim), 346, 349 (2013).

11. X.H. Liu, J.Q. Weng and C.X. Tan, Asian J. Chem., 23, 4064 (2011).

12. X.H. Liu, J.Q. Weng, C.X. Tan, B.L. Wang and Z.M. Li, Asian J. Chem., 23, 4031 (2011). 